\title{
CYP2D6 phenotype explains reported yohimbine concentrations in four severe acute intoxications
}

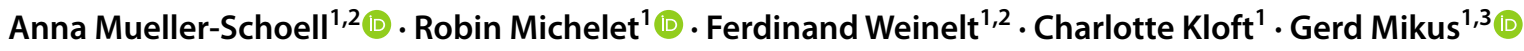

Received: 13 April 2021 / Accepted: 17 May 2021 / Published online: 24 May 2021

(c) The Author(s) 2021

\begin{abstract}
The indole alkaloid yohimbine is an alpha-2 receptor antagonist used for its sympathomimetic effects. Several cases of yohimbine intoxication have been reported and the most recent one involved four individuals taking a yohimbine-containing drug powder. All individuals developed severe intoxication symptoms and were admitted to the hospital. Even though all individuals were assumed to have taken the same dose of the drug powder, toxicology analyses revealed yohimbine blood concentrations of $249-5631 \mathrm{ng} / \mathrm{mL}$, amounting to a 22 -fold difference. The reason for this high variability remained to be elucidated. We used recently reported knowledge on the metabolism of yohimbine together with state-of-the art nonlinear mixed-effects modelling and simulation and show that a patient's cytochrome P450 2D6 (CYP2D6) phenotype can explain the large differences observed in the measured concentration after intake of the same yohimbine dose. Our findings can be used both for the identification of safe doses in therapeutic use of yohimbine and for an explanation of individual cases of overdosing.
\end{abstract}

Keywords Yohimbine $\cdot$ CYP2D6 $\cdot$ Pharmacokinetics $\cdot$ Clearance $\cdot$ Modelling \& simulation $\cdot$ Pharmacometrics

Yohimbine is a plant-derived indole alkaloid and alpha-2 receptor antagonist (Swann et al. 2013), historically used for erectile dysfunction (Wishart et al. 2018) but also abused in the bodybuilding community (Drevin et al. 2020). Recently, it has received increased attention as a promising compound for the microdose-based identification of a patient's cytochrome P450 (CYP) metaboliser status (Vay et al. 2020). Over the years, several cases of yohimbine intoxication have been reported (Giampreti et al. 2009; Anderson et al. 2013; Gicquel et al. 2016; Drevin et al. 2020). The most recent case report on four individuals intoxicated with yohimbine described four individuals with severe intoxication symptoms leading to hospitalisation approximately $10 \mathrm{~h}$

Gerd Mikus

gerd.mikus@fu-berlin.de

1 Department of Clinical Pharmacy and Biochemistry, Institute of Pharmacy, Freie Universitaet Berlin, Kelchstr. 31, 12169 Berlin, Germany

2 Graduate Research Training Program PharMetrX, Berlin/Potsdam, Germany

3 Department of Clinical Pharmacology and Pharmacoepidemiology, University Hospital Heidelberg, Im Neuenheimer Feld 410, 69120 Heidelberg, Germany after taking a drug powder containing yohimbine (Zhu et al. 2021). Of the four individuals, three recovered after treatment and one died before arrival at the hospital. The three recovered individuals took $4-5 \mathrm{~g}$ of the drug powder. While no amount could be reported for the deceased person, it is likely, and we assume that the same amount was taken. From every person, a blood sample was obtained between 10 and $11 \mathrm{~h}$ after yohimbine intake. The concentrations measured via LC-MS/MS were $459,249,301$, and $5631 \mathrm{ng} / \mathrm{mL}$ with the highest concentration measured in the deceased person. The authors highlighted the potential risk for yohimbine overdosing and we appreciate their important contribution to defining a therapeutic window for yohimbine. However, while the authors focused on the high interindividual variability in bioavailability as a possible explanation for the very different concentrations measured between 10 and $11 \mathrm{~h}$ after intake of yohimbine, we think that newly emerged knowledge on its pharmacokinetics and metabolism (Vay et al. 2020) together with pharmacokinetic simulations can further elucidate reasons for the observed high yohimbine concentrations.

There are only a few publications on the pharmacokinetics of yohimbine, whose bioavailability is highly variable (Guthrie et al. 1990) and elimination has been poorly 
characterised for a long time. Recently published new data identified the highly polymorphic CYP2D6 as a major contributor to the clearance of yohimbine (Vay et al. 2020). In this work, the pharmacokinetics of a single oral dose of $5 \mathrm{mg}$ yohimbine was characterised in CYP2D6 genotyped Caucasian volunteers. In line with the large differences in CYP2D6 metabolic activity, the apparent oral clearance varied substantially by more than 600 -fold $(25-15,863 \mathrm{~mL} / \mathrm{min})$ and terminal elimination half-life ranged from 0.5 to $7.6 \mathrm{~h}$. It is known that the CYP2D6 activity distribution is different between Caucasian and Chinese individuals. While East Asians show a lower frequency of individuals with no enzymatic activity, the frequency of decreased enzyme activity is much higher compared to Caucasians (Bertilsson et al. 1992; Huddart et al. 2019; Nofziger et al. 2020, Mueller-Schoell et al. 2021). In combination with the high dose, this reduced metabolic activity could have resulted in the highly toxic yohimbine concentrations in the four Chinese individuals.

To explore this hypothesis, we used the pharmacokinetic data published (Vay et al. 2020) to develop a nonlinear mixed-effects pharmacokinetic model of yohimbine. In short, a two-compartmental model with first-order absorption and linear elimination was fitted to the data reported by Vay et al. using the software NONMEM v. 7.4 (ICON Development Solutions, Ellicott City, MD, USA). The interindividual variability on the yohimbine clearance was largely explained by CYP2D6 activity, leading to a reduction from 1143 to $43.9 \mathrm{CV} \%$ after inclusion of genotype-derived phenotype as a covariate. The individual yohimbine clearance was then estimated using maximum-a-posteriori likelihood (MAP) estimation with the developed yohimbine model as prior. Subsequently, the individual model was simulated using mrgsolve (v.0.10.1) in R/Rstudio (v. 3.6.3/1.3.959). Given the information known about the four intoxicated patients (i.e., approximate amount of drug taken, approximate time of blood sampling, and measured yohimbine concentrations), the developed model was then used to estimate each of the intoxicated patients' yohimbine clearance and most likely CYP2D6 phenotype, considering the yohimbine clearances observed in patients of different phenotypes in the study by Vay et al. For the four intoxicated patients, the time points of blood sampling were set to $10.5 \mathrm{~h}$ since only an approximate value was given. Furthermore, the intake of $5 \mathrm{mg}$ of yohimbine was assumed for all individuals.

The measured and model-predicted concentrations at approximately $10.5 \mathrm{~h}$ after intake together with the predicted apparent clearances and half-lives are reported in Table 1 and the simulated yohimbine concentration-time profiles along with the measured concentrations are shown in Fig. 1.

According to the model predictions, extensive metabolisers exhibit a yohimbine CL/F greater than $3000 \mathrm{~mL} /$ min whereas poor metabolisers exhibit a yohimbine CL/F below $100 \mathrm{~mL} / \mathrm{min}$. Based on the estimated clearance values, all four patients were phenotypic CYP2D6 intermediate metabolisers with decreased metabolic activity.

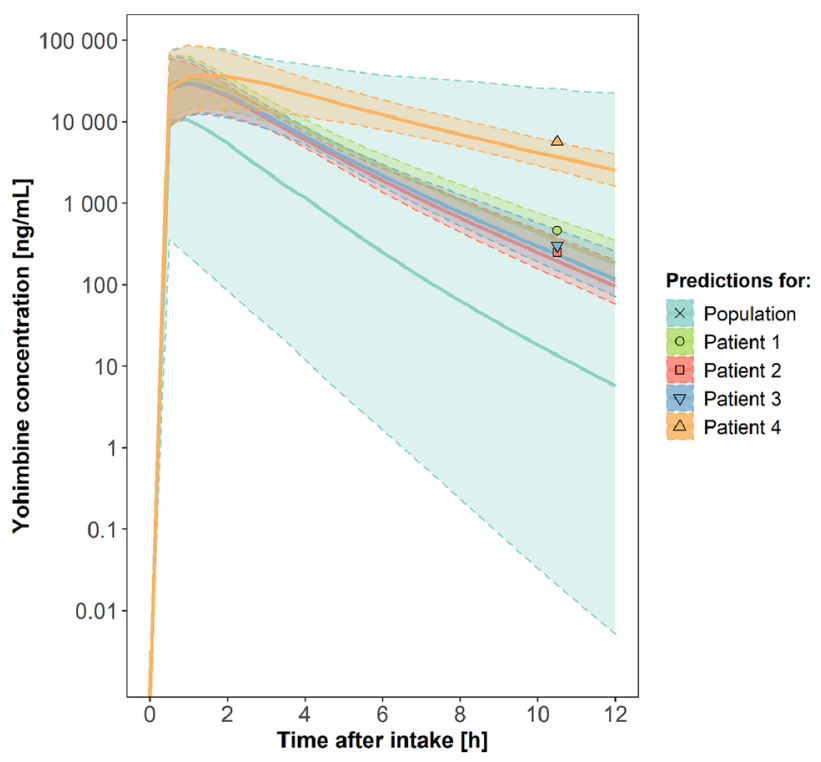

Fig. 1 Model-predicted and observed concentrations after a single administration of $5 \mathrm{~g}$ yohimbine. Model predictions based on stochastic simulations $(n=1000)$ of the prior (population) or posterior (individual patient's) variability distribution of the yohimbine pharmacokinetic model. Solid lines: median predicted concentrations, dotted lines and coloured shades: $90 \%$ prediction intervals
Table 1 Pharmacokinetic simulation results for yohimbine in the four intoxication cases, assuming a $5 \mathrm{~g}$ dose and a sampling time of $10.5 \mathrm{~h}$

\begin{tabular}{lllll}
\hline & Case 1 & Case 2 & Case 3 & Case 4 \\
\hline $\begin{array}{l}\text { Measured } \mathrm{C}_{10.5 \mathrm{~h}}[\mathrm{ng} / \mathrm{mL}] \\
\text { Simulation }\end{array}$ & 459 & 249 & 301 & 5631 \\
$\mathrm{CL} / \mathrm{F}[\mathrm{mL} / \mathrm{min}]$ & & & & \\
$\mathrm{t}_{1 / 2}[\mathrm{~h}]$ & 822.3 & 1013.2 & 927.3 & 403.1 \\
Predicted $\mathrm{C}_{10.5 \mathrm{~h}}$ Median $(90 \% \mathrm{PI})[\mathrm{ng} / \mathrm{mL}]$ & 0.74 & 0.64 & 0.69 & 1.80 \\
& $349.5(226.8-$ & $196.5(121.9-$ & $240.4(147.0-$ & 3675.0 \\
& $648.2)$ & $430.8)$ & $466.8)$ & $(2478.1-$ \\
Median predicted $\mathrm{C}_{\max }[\mathrm{ng} / \mathrm{mL}]$ & 34,148 & 30,328 & 30,160 & 41,299 \\
\hline
\end{tabular}

$C_{10.5 \mathrm{~h}}$ concentration at $10.5 \mathrm{~h}$ after intake; $C L / F$ apparent clearance; $t_{l / 2}$ half-life; $P I$ prediction interval; $C_{\max }$ maximum concentration 
In addition, there might have been some degree of autoinhibition which has been reported for yohimbine before (Vay et al. 2020). Yet, since very high doses such as $5 \mathrm{~g}$ have never been investigated before, the degree of autoinhibition and its contribution to the observed decreased clearance is unknown and needs further evaluation.

There are several limitations associated with our study: first, while we assumed that all patients ingested $5 \mathrm{~g}$ of yohimbine, the precise amount of yohimbine in the drug powder was not known. The assumed dose of $5 \mathrm{~g}$ yohimbine supposes a purity of $100 \%$ in the drug powder, thus, it is possible that a lower amount was ingested which, given the measured concentrations, would result in even lower estimated yohimbine clearances. The dose ingested by patient four is unknown. Considering that this patient died after drug powder intake and he had a more than tenfold higher concentration compared to the other three patients, either the ingested dose was much higher or he had a lower CYP2D6 activity or a combination of both. Second, as the CYP2D6 genotypes of the four patients were not determined, we were not able to test the validity of our model predictions. While the ability of the model to well capture the observed concentrations supports its performance, the feasibility of estimating an individual's CYP2D6 phenotype based on measured yohimbine concentrations should be confirmed with an independent dataset incorporating measured yohimbine concentrations and the CYP2D6 genotype and phenotype in the future.

In conclusion, the CYP2D6 metabolic activity plays a key role in the metabolism of yohimbine and especially individuals with decreased activity are at risk for overdosing/toxic concentrations. The usual therapeutic dose of $15-30 \mathrm{mg}$ is sometimes exceeded and warnings about potentially dangerous side effects (including death) are stated without data supporting it (WebMD). Because the reported amounts taken in the case report were certainly excessively high, the outcome was not preventable. Yet, if yohimbine is used therapeutically, the consideration of an individual's CYP2D6 phenotype/metabolic activity will reduce the risk for toxic concentrations and increase drug safety. Determining a patient's CYP2D6 activity by phenotyping before treatment initiation can minimise the risk for individual overdosing in the therapeutic setting. Leveraging prior pharmacokinetic knowledge with forensic (or toxicology) drug monitoring in a modelling and simulation framework seems an appealing approach in elucidating individual overdosing if a CYP2D6 activity measurement is not available.

Funding Open Access funding enabled and organized by Projekt DEAL.
Data availability Not applicable.

Code availability The NONMEM model code for the nonlinear mixedeffects model is available on reasonable request from the corresponding author.

\section{Declarations}

Conflict of interest C.K. reports grants from an industry consortium (AbbVie Deutschland GmbH and Co. KG, AstraZeneca Ltd, Boehringer Ingelheim Pharma GmbH \& Co. KG, Grünenthal GmbH, F. Hoffmann-La Roche Ltd, Merck KGaA and Sanofi) for the PharMetrX PhD program and from the Federal Ministry of Education and Research as well as the European Commission for the Innovative Medicines Initiative-Joint Undertaking, all outside the submitted work. The other authors declare that they have no conflict of interest.

Ethical standards The manuscript does not contain clinical studies or patient data.

Ethics approval Not applicable.

Consent to participate Not applicable.

Consent for publication Not applicable.

Open Access This article is licensed under a Creative Commons Attribution 4.0 International License, which permits use, sharing, adaptation, distribution and reproduction in any medium or format, as long as you give appropriate credit to the original author(s) and the source, provide a link to the Creative Commons licence, and indicate if changes were made. The images or other third party material in this article are included in the article's Creative Commons licence, unless indicated otherwise in a credit line to the material. If material is not included in the article's Creative Commons licence and your intended use is not permitted by statutory regulation or exceeds the permitted use, you will need to obtain permission directly from the copyright holder. To view a copy of this licence, visit http://creativecommons.org/licenses/by/4.0/.

\section{References}

Anderson C, Anderson D, Harre N, Wade N (2013) Case study: two fatal case reports of acute yohimbine intoxication. J Anal Toxicol 37:611-614. https://doi.org/10.1093/jat/bkt057

Bertilsson L, Lou YQ, Du YL et al (1992) Pronounced differences between native Chinese and Swedish populations in the polymorphic hydroxylations of debrisoquin and S-mephenytoin. Clin Pharmacol Ther 51:388-397. https://doi.org/10.1038/clpt. 1992.38

Drevin G, Palayer M, Compagnon P et al (2020) A fatal case report of acute yohimbine intoxication. Forensic Toxicol 38:287-291. https://doi.org/10.1007/s11419-019-00512-y

Giampreti A, Lonati D, Locatelli C et al (2009) Acute neurotoxicity after yohimbine ingestion by a body builder. Clin Toxicol 47:827-829. https://doi.org/10.1080/15563650903081601

Gicquel T, Hugbart C, Le Devehat F et al (2016) Death related to consumption of Rauvolfia sp. powder mislabeled as Tabernanthe iboga. Forensic Sci Int 266:e38-e42. https://doi.org/10.1016/j. forsciint.2016.06.014 
Guthrie SK, Hariharan M, Grunhaus LJ (1990) Yohimbine bioavailability in humans. Eur J Clin Pharmacol 39:409-411. https:// doi.org/10.1007/BF00315421

Huddart R, Fohner AE, Whirl-Carrillo M et al (2019) Standardized biogeographic grouping system for annotating populations in pharmacogenetic research. Clin Pharmacol Ther 105:12561262. https://doi.org/10.1002/cpt.1322

Mueller-Schoell A, Michelet R, Klopp-Schulze L, van Dyk M, Mürdter TE, Schwab M, Joerger M, Huisinga W, Mikus G, Kloft C (2021) Computational treatment simulations to assess the need for personalized tamoxifen dosing in breast cancer patients of different biogeographical groups. Cancers 13:2432. https://doi.org/10. 3390/cancers 13102432

Nofziger C, Turner AJ, Sangkuhl K et al (2020) PharmVar GeneFocus: CYP2D6. Clin Pharmacol Ther 107:154-170. https://doi. org/10.1002/cpt.1643

Swann AC, Lijffijt M, Lane SD et al (2013) Norepinephrine and impulsivity: effects of acute yohimbine. Psychopharmacology 229:83-94. https://doi.org/10.1007/s00213-013-3088-7
Vay M, Meyer MJ, Blank A et al (2020) Oral Yohimbine as a new probe drug to predict CYP2D6 activity: results of a fixedsequence phase I trial. Clin Pharmacokinet 59:927-939. https:// doi.org/10.1007/s40262-020-00862-6

Wishart DS, Feunang YD, Guo AC et al (2018) DrugBank 5.0: a major update to the DrugBank database for 2018. Nucleic Acids Res 46:D1074-D1082. https://doi.org/10.1093/nar/gkx1037

WebMD Yohimbe. https://www.webmd.com/vitamins/ai/ingredient mono-759/yohimbe. (Accessed 5 Mar 2021)

Zhu L, Han X, Zhu J et al (2021) Severe acute intoxication with yohimbine: four simultaneous poisoning cases. Forensic Sci Int. https:// doi.org/10.1016/j.forsciint.2021.110705

Publisher's Note Springer Nature remains neutral with regard to jurisdictional claims in published maps and institutional affiliations. 